\title{
The cost of wobble translation in fungal mitochondrial genomes: integration of two traditional hypotheses
} Xuhua Xia ${ }^{1,2}$

Address: ${ }^{1}$ Department of Biology and Center for Advanced Research in Environmental Genomics, University of Ottawa 30 Marie Curie, Ottawa, K1N 6N5, Canada and 2Ottawa Institute of Systems Biology, University of Ottawa, Ottawa, Canada

Email: Xuhua Xia - xxia@uottawa.ca

Published: 19 July 2008

BMC Evolutionary Biology 2008, 8:2 I I doi: I0.1 I86/I47I-2I48-8-2I I

This article is available from: http://www.biomedcentral.com//47|-2|48/8/2I I

(c) 2008 Xia; licensee BioMed Central Ltd.

This is an Open Access article distributed under the terms of the Creative Commons Attribution License (http://creativecommons.org/licenses/by/2.0), which permits unrestricted use, distribution, and reproduction in any medium, provided the original work is properly cited.
Received: 27 February 2008

Accepted: 19 July 2008

ution, and reproduction in any medium, provided the original work is properly cited.

\begin{abstract}
Background: Fungal and animal mitochondrial genomes typically have one tRNA for each synonymous codon family. The codon-anticodon adaptation hypothesis predicts that the wobble nucleotide of a tRNA anticodon should evolve towards maximizing Watson-Crick base pairing with the most frequently used codon within each synonymous codon family, whereas the wobble versatility hypothesis argues that the nucleotide at the wobble site should be occupied by a nucleotide most versatile in wobble pairing, i.e., the tRNA wobble nucleotide should be G for NNY codon families, and $U$ for NNR and NNN codon families (where $Y$ stands for $C$ or $U, R$ for $A$ or $\mathrm{G}$ and $\mathrm{N}$ for any nucleotide).
\end{abstract}

Results: We here integrate these two traditional hypotheses on tRNA anticodons into a unified model based on an analysis of the wobble costs associated with different wobble base pairs. This novel approach allows the relative cost of wobble pairing to be qualitatively evaluated. A comprehensive study of 36 fungal genomes suggests very different costs between two kinds of $U: G$ wobble pairs, i.e., ( $I$ ) between a $G$ at the wobble site of a tRNA anticodon and a $U$ at the third codon position (designated $M_{U 3: G}$ ) and (2) between a $U$ at the wobble site of a tRNA anticodon and $a \mathrm{G}$ at the third codon position (designated $\mathrm{M}_{\mathrm{G} 3: U}$ ).

Conclusion: In general, $M_{U 3: G}$ is much smaller than $M_{\mathrm{G} 3: U}$, suggesting no selection against $U$-ending codons in NNY codon families with a wobble $G$ in the tRNA anticodon but strong selection against G-ending codons in NNR codon families with a wobble $U$ at the tRNA anticodon. This finding resolves several puzzling observations in fungal genomics and corroborates previous studies showing that U3:G wobble is energetically more favorable than G3:U wobble.

\section{Background}

The wobble versatility hypothesis [1-6], abbreviated as WVH, states that the wobble site of tRNA anticodon should have G for NNY codons (where Y stands for C or $\mathrm{U}$ and $\mathrm{N}$ for any nucleotide) because $\mathrm{G}$ can pair with both $\mathrm{C}$ and $\mathrm{U}$ in RNA, and should have $U$ for NNR to pair with both $\mathrm{A}$ and $\mathrm{G}$. For NNN codon families, the wobble site should be $U$ because $U$ is known to be the most versatile in wobble-pairing [7-12]. In contrast, the codon-anticodon adaptation hypothesis, or CAAH for short, invokes the codon usage bias as a determining factor, i.e., the wobble site of tRNA anticodon should co-evolve with codon usage so that the nucleotide in the wobble site of tRNA anticodon should match the most abundant codon in a synonymous codon family [6,13-15]. The association between the major codon and the anticodon of the most 
abundant tRNA has been documented in Escherichia coli $[16,17]$, Saccharomyces cerevisiae [18], and other species and organelles [15,19-22].

Here we develop a general hypothesis of codon-anticodon adaptation based on an analysis of wobble costs, and derive its predictions that can be tested by genomic data. The wobble cost may be viewed as reduction in decoding efficiency and accuracy because such reduction would be selected against over evolutionary time. We will refer to this new general hypothesis based on wobble cost as WCH (for wobble cost hypothesis). The two traditional hypotheses, CAAH and WVH, will be shown to be special forms of WCH.

Following the shorthand notation of Ogle et al. [23], I designate the translation cost through wobble base-pairing between nucleotide $i$ at the third codon position of a codon and the nucleotide $\mathrm{j}$ at the wobble site of tRNA anticodon as $\mathrm{M}_{\mathrm{i} 3: \mathrm{j}}$ (where $\mathrm{M}$ is for wobble cost. The letter $\mathrm{C}$ would be more suitable to represent cost but it may confuse with the nucleotide $C$ ). We assume $M_{i 3: j}=0$ if nucleotides $\mathrm{i}$ and $\mathrm{j}$ form Watson-Crick base pairing. The reason for this assumption is that $C: G$ and $A: U$ pairs have not been found to contribute to ribosomal stalling (which reduces translation efficiency) or amino acid mis-incorporation (which reduces translation accuracy) although almost all non-Watson-Crick base pairings have been shown to reduce translation efficiency and accuracy. We define $\mathrm{M}_{\mathrm{Y} 3: \mathrm{U}}$ as the wobble cost between a wobble $\mathrm{U}$ at the tRNA anticodon and a $\mathrm{C}$ or $\mathrm{U}$ at the third codon position. For simplicity, we also assume $\mathrm{M}_{\mathrm{A} 3: \mathrm{A}}=\mathrm{M}_{\mathrm{C} 3: \mathrm{A}}=\mathrm{M}_{\mathrm{G} 3: \mathrm{A}}=$ $\mathrm{M}_{\mathrm{A} 3: \mathrm{C}}=\mathrm{M}_{\mathrm{C} 3: \mathrm{C}}=\mathrm{M}_{\mathrm{U} 3: \mathrm{C}}=\mathrm{M}_{\mathrm{A} 3: \mathrm{G}}=\mathrm{M}_{\mathrm{G} 3: \mathrm{G}}=\mathrm{M}_{\mathrm{O}}$ (where the subscript $\mathrm{O}$ is for "other", i.e., $\mathrm{M}_{\mathrm{O}}$ is the wobble cost between base pairs that are not Watson-Crick base pairs, not U3:G or G3:U base pairs and not Y3:U pairs). In general, $\mathrm{M}_{\mathrm{U} 3: \mathrm{G}}$ and $\mathrm{M}_{\mathrm{G} 3: \mathrm{U}}$ are expected to be smaller than $\mathrm{M}_{\mathrm{C} 3: \mathrm{U},}, \mathrm{M}_{\mathrm{U} 3: \mathrm{U}}$, and $\mathrm{M}_{\mathrm{O}}$ because $\mathrm{G}$ and $\mathrm{U}$ can base-pair in RNA, $M_{Y 3: U}$ is expected to be smaller than $M_{O}$ because a wobble $U$ at tRNA anticodon is known to be the most versatile in wobble-pairing [7-12].

A classical study of U:G wobble pairs [24] suggests a preference for the $U$ being at the $3^{\prime}$ end rather than at the $5^{\prime}$ end, which implies that U3:G wobble pair is energetically more favorable than G3:U wobble pair [25]. Subsequent studies have shown that, while the U3:G wobble pair occurs on the ribosome, the unmodified G3:U wobble pair does not $[2,23,26]$. These findings suggest that $M_{U 3: G}$ may be smaller than $\mathrm{M}_{\mathrm{G} 3: \mathrm{U}^{\prime}}$ although its generality is unknown.

\section{Two-fold NNY and NNR codon families}

First consider the NNY codon family where $\mathrm{Y}$ is either $\mathrm{C}$ or $\mathrm{U}$. Designate the number of C-ending and U-ending syn- onymous codons by $\mathrm{N}_{\mathrm{C}}$ and $\mathrm{N}_{\mathrm{U}}$, respectively, and the total cost of wobble pairing as $\mathrm{M}_{\mathrm{wG}}$ when the wobble site of the anticodon is $\mathrm{G}$, and as $\mathrm{M}_{\mathrm{wA}}$ when the wobble nucleotide is $\mathrm{A}$ (we do not need to consider the case when the wobble site is $\mathrm{U}$ or $\mathrm{C}$ for NNY codon families because such cases have never been observed and because a tRNA with a wobble $\mathrm{U}$ or $\mathrm{C}$ to translate NNC and NNU codons is against physiochemical reasons). We now express the total cost $\mathrm{M}_{\mathrm{wG}}$ and $\mathrm{M}_{\mathrm{wA}}$ as

$$
\begin{aligned}
& M_{w G}=N_{U} M_{U 3: G} \\
& M_{w A}=N_{C} M_{C 3: A}=N_{C} M_{O}
\end{aligned}
$$

Note that $\mathrm{M}_{\mathrm{C} 3: \mathrm{G}}=\mathrm{M}_{\mathrm{U} 3: \mathrm{A}}=0$ according to our definition. The dependence of $\mathrm{M}_{\mathrm{wG}}$ and $\mathrm{M}_{\mathrm{wA}}$ on the relative frequencies of $\mathrm{N}_{\mathrm{C}^{\prime}}$ expressed as proportion of $\mathrm{C}$-ending codons in the NNY codon family $\left(\mathrm{P}_{\mathrm{C}}\right)$, is graphically shown in Fig. 1 , with $\mathrm{M}_{\mathrm{O}}$ assumed to equal $2 \bullet ; \mathrm{M}_{\mathrm{U} 3: \mathrm{G}}$. The condition for $M_{w G}=M_{w A}$, i.e., when the wobble site of the tRNA anticodon can take either $\mathrm{G}$ or A without a fitness differential, is

$$
\frac{M_{U 3: G}}{M_{O}}=\frac{N_{C}}{N_{U}}=\frac{P_{C}}{1-P_{C}}
$$

In the scenario in Fig. 1 with $\mathrm{M}_{\mathrm{O}}=2 \mathrm{M}_{\mathrm{U}: \mathrm{G}^{\prime}}$ the condition for $M_{w G}=M_{w A}$ is $P_{C}=1 / 3$. Thus, when $P_{C}$ drifts above $1 / 3$, it would become more beneficial (less costly) to have a $\mathrm{G}$ at the wobble nucleotide of tRNA anticodon. However, when PC drifts below $1 / 3$, then a wobble A would be more advantageous and should be favored by natural selection. Naturally, if $\mathrm{M}_{\mathrm{U}: \mathrm{G}}=0$, then we should always

have a wobble $\mathrm{G}$ at the tRNA anticodon regardless of codon usage.

Now we consider three special cases with reference to Fig. 1 and the two traditional hypotheses, CAAH and WVH. First, if $\mathrm{N}_{\mathrm{C}}=\mathrm{N}_{\mathrm{U}}=\mathrm{N} / 2$, then CAAH, which predicts that the wobble nucleotide of tRNA anticodon should form Watson-Crick base pair with the most abundant codon, has no prediction at all because the two codons are equally abundant. In contrast, if we assume $\mathrm{M}_{\mathrm{U}: \mathrm{G}}<\mathrm{M}_{\mathrm{O}}$ as is depicted in Fig. 1, we have $\mathrm{M}_{\mathrm{wG}}<\mathrm{M}_{\mathrm{wA}}$ and WCH predicts that the anticodon wobble site should be a G. This is the same prediction as $\mathrm{WVH}$.

Second, if $\mathrm{N}_{\mathrm{C}}>\mathrm{N}_{\mathrm{U}}$, then $\mathrm{M}_{\mathrm{wG}}<\mathrm{M}_{\mathrm{wA}}$ assuming $\mathrm{M}_{\mathrm{U3}: \mathrm{G}} \leq \mathrm{M}_{\mathrm{O}}$, and WCH predicts that the anticodon wobble site should be occupied by a G. This prediction is shared by both CAAH and WVH.

Third, when $\mathrm{N}_{\mathrm{C}}<\mathrm{N}_{\mathrm{U}}$ but $\mathrm{N}_{\mathrm{C}}>\mathrm{N}_{\mathrm{U}} \cdot \mathrm{M}_{\mathrm{U3:G}} / \mathrm{M}_{\mathrm{O}}$, then WCH will still predict a $G$ at the wobble nucleotide of tRNA anticodon because $\mathrm{M}_{\mathrm{wG}}$ is still smaller than $\mathrm{M}_{\mathrm{wA}}$. Take the scenario in Fig. 1 for example, when $\mathrm{N}_{\mathrm{C}}<\mathrm{N}_{\mathrm{U}}$ but $\mathrm{P}_{\mathrm{C}}>1 / 3$ 


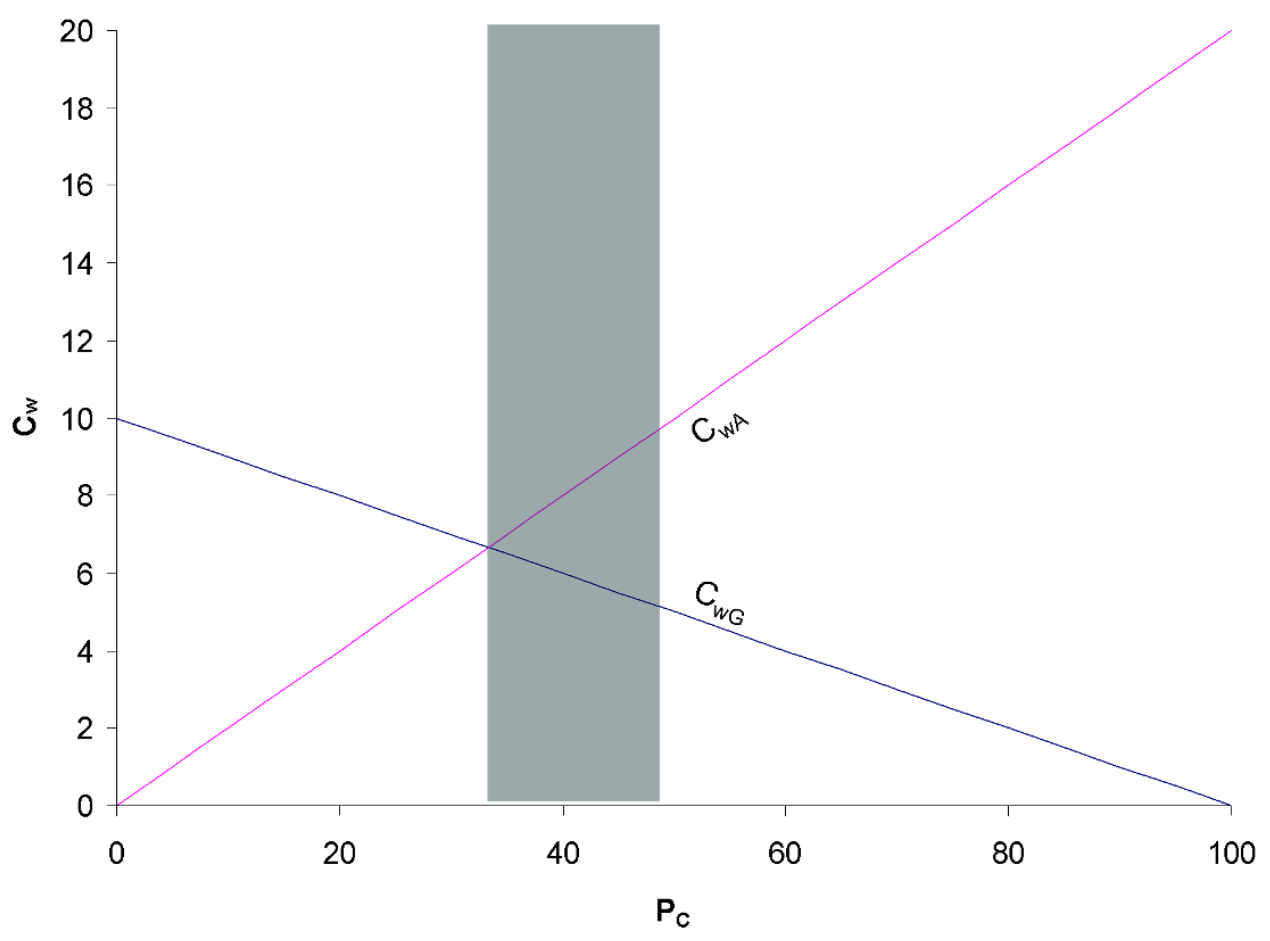

\section{Figure I}

Conceptual illustration of the dependence of wobbling cost involving a $G$ or an $A$ at the wobble site of tRNA anticodon ( $M_{w G}$ and $\left.M_{w A}\right)$ on the proportion of $C$-ending codons $\left(P_{C}\right)$ in an NNY codon family, with $M_{C 3: A}=2 M_{U 3: G}$. The shaded area corresponds to $P_{C}$ smaller than $I / 2$ but larger than $I / 3$.

(which correspond to the shaded area in Fig. 1), we have $M_{\mathrm{wG}}<\mathrm{M}_{\mathrm{wA}}$ (Fig. 1), so natural selection should favor a $\mathrm{G}$ at the wobble site of tRNA anticodon. WVH happens to have the same prediction. However, CAAH will predict an $\mathrm{A}$ at the wobble site of tRNA anticodon if U-ending codons are more abundant than C-ending codons. This is in contrast to $\mathrm{WVH}$, i.e. CAAH is inapplicable when $\mathrm{P}_{\mathrm{C}}$ is within the shaded range in Fig. 1.

Fourth, when $\mathrm{N}_{\mathrm{C}} \ll \mathrm{N}_{\mathrm{U}}$, especially in the extreme case when $N_{C}=0$, then Eq. (1) is reduced to $M_{w G}=N_{U} \cdot M_{U 3: G}$ and $\mathrm{M}_{\mathrm{wA}}=0$. Because now $\mathrm{M}_{\mathrm{wG}}>\mathrm{M}_{\mathrm{wA}^{\prime}}$ WCH predicts an $A$ at the anticodon wobble site. In this case, WVH would still predict a $G$ at the wobble site because it ignores the codon frequencies (i.e., it ignores the relative magnitude of $\mathrm{N}_{\mathrm{C}}$ and $\mathrm{N}_{\mathrm{U}}$ ), but CAAH would predict an $\mathrm{A}$ at the wobble site, which is the same prediction as $\mathrm{WCH}$. Only in this particular case when $\mathrm{N}_{\mathrm{C}}<<\mathrm{N}_{\mathrm{U}}$ can CAAH and WVH be clearly differentiated. As is depicted in Fig. 1, the chance for CAAH to be supported decreases as $\mathrm{M}_{\mathrm{U} 3: \mathrm{G}}$ decreases relative to $M_{O}$. CAAH would have little chance to be unambiguously supported if $\mathrm{M}_{\mathrm{U} 3: \mathrm{G}}$ is close to 0 . Thus, as long as $\mathrm{M}_{\mathrm{U3:G}}$ is small relative to $\mathrm{M}_{\mathrm{O}}$, we should expect CAAH to be less supported than WVH. A recent study shows that CAAH indeed receives much less support than WVH when both were tested against fungal mitochondrial genomic data [27].

The simple Eq. (1) offers a way to assess relative costs of different wobble pairs. For example, if nature has chosen a $G$ at the wobble site of the tRNA anticodon, then we may infer that $\mathrm{M}_{\mathrm{wG}}<\mathrm{M}_{\mathrm{wA}}$. So, from Eq. (1), we have

$$
\begin{aligned}
& N_{U} M_{U 3: G}<N_{C} M_{O} \\
& \frac{M_{U 3: G}}{M_{O}}<\frac{N_{C}}{N_{U}}
\end{aligned}
$$

Suppose we have an NNY codon family translated by a tRNA with a $G$ at the wobble site of the anticodon. If $\mathrm{M}_{\mathrm{U} 3: \mathrm{G}}$ is large, then there would be strong selection favoring C-ending codons against $\mathrm{U}$ ending codons. So the ratio of $\mathrm{N}_{\mathrm{C}} / \mathrm{N}_{\mathrm{U}}$ may be very large. In such a case the estimated $\mathrm{M}_{\mathrm{U} 3: \mathrm{G}} / \mathrm{M}_{\mathrm{O}}$ ratio, being smaller than a very large value, is not informative. However, in different codon families, mutation pressure may allow $\mathrm{N}_{\mathrm{U}}$ to drift up relative to $\mathrm{N}_{\mathrm{C}}$. Suppose we have three NNY codon families each translated by a tRNA with a $G$ at the anticodon wobble site. If the $\mathrm{N}_{\mathrm{C}} / \mathrm{N}_{\mathrm{U}}$ ratio for the three $\mathrm{NNY}$ codon families are 100/2,80/100 and 200/20, respectively, we may infer that $\mathrm{M}_{\mathrm{U} 3: \mathrm{G}} / \mathrm{M}_{\mathrm{O}}<80 / 100$ (because it is the most pre- 
cise). Alternatively, with many $\mathrm{N}_{\mathrm{C}} / \mathrm{N}_{\mathrm{U}}$ ratios from many NNY codon families translated by tRNA with a $G$ at its anticodon wobble site, we may compute the lower 95\% confidence limit of the $\mathrm{N}_{\mathrm{C}} / \mathrm{N}_{\mathrm{U}}$ ratio ( $\mathrm{LCL}_{95 . \mathrm{G}}$ where the subscript $G$ indicates tRNA with a $G$ at the anticodon wobble site) and infer that $\mathrm{M}_{\mathrm{U3:G}} / \mathrm{M}_{\mathrm{C3:A}}<\mathrm{LCL}_{95 . \mathrm{G}}$.

If we always have very large $\mathrm{N}_{\mathrm{C}} / \mathrm{N}_{\mathrm{U}}$ ratios, we may infer that selection against U-ending codons must be strong, with little chance for mutation to elevate $\mathrm{N}_{\mathrm{U}}$. This is a strong indication of a large $\mathrm{M}_{\mathrm{U} 3: \mathrm{G}}$. Along the same line of reasoning, we may infer that $\mathrm{M}_{\mathrm{U3:G}}$ is very small if $\mathrm{N}_{\mathrm{U}}$ can often as large as, or even larger than, $\mathrm{N}_{\mathrm{C}}$.

Similarly, if nature has chosen an A at the wobble site of tRNA anticodon, then we may infer $\mathrm{M}_{\mathrm{wG}}>\mathrm{M}_{\mathrm{wA}^{\prime}}$ so

$$
\begin{aligned}
& N_{U} M_{U 3: G}>N_{C} M_{O} \\
& \frac{M_{U 3: G}}{M_{O}}>\frac{N_{C}}{N_{U}}
\end{aligned}
$$

We can apply exactly the same rationale for the NNR codon family leading to parallel conclusions. For example, if nature has chosen a $U$ at the wobble site of the tRNA anticodon, then we may infer that $\mathrm{M}_{\mathrm{wU}}<\mathrm{M}_{\mathrm{wC}}$, so that

$$
\frac{M_{G 3: U}}{M_{O}}<\frac{N_{A}}{N_{G}}
$$

Similarly, if $\mathrm{M}_{\mathrm{G} 3: \mathrm{U}}$ is very large, then there should be strong selection against G-ending codons in favor of Aending codons. This will produce large $\mathrm{N}_{\mathrm{A}} / \mathrm{N}_{\mathrm{G}}$ ratios. In contrast, a large $\mathrm{N}_{\mathrm{G}}$ comparable to $\mathrm{N}_{\mathrm{A}}$ indicates a very small $\mathrm{M}_{\mathrm{G} 3: \mathrm{U}}$ cost.

Given previous studies indicating that $\mathrm{U} 3: \mathrm{G}$ is energetically much more favorable than G3:U [2,23-26], we should expect $\mathrm{M}_{\mathrm{U} 3: \mathrm{G}}<\mathrm{M}_{\mathrm{G} 3: \mathrm{U}}$. The reasoning above paves the way for us to test whether this is generally true among the genetically diverse fungal species.

\section{Three-fold AUH (Isoleucine) codons}

Designate codons ending with $\mathrm{A}, \mathrm{C}$, and $\mathrm{U}$ as $\mathrm{N}_{\mathrm{A}^{\prime}} \mathrm{N}_{\mathrm{C}}$ and $\mathrm{N}_{\mathrm{U}}$, respectively. The wobble cost of having an $\mathrm{A}, \mathrm{G}, \mathrm{C}$, or $\mathrm{U}$ at the wobble site of tRNA anticodon is

$$
\begin{aligned}
& M_{w A}=N_{A} M_{A 3: A}+N_{C} M_{C 3: A}=\left(N_{A}+N_{C}\right) M_{O} \\
& M_{w G}=N_{A} M_{A 3: G}+N_{U} M_{U 3: G}=N_{A} M_{O}+N_{U} M_{U 3: G} \\
& M_{w C}=N_{A} M_{A 3: C}+N_{C} M_{C 3: C}+N_{U} M_{U 3: C}=\left(N_{A}+N_{C}\right) M_{O}+N_{U} M_{U 3: C} \\
& M_{w U}=N_{C} M_{C 3: U}+N_{U} M_{U 3: U}=\left(N_{C}+N_{U}\right) M_{Y 3: U}
\end{aligned}
$$

It is obvious that $M_{w C}$ is always greater than $M_{w A^{\prime}}$ so we should never find a $\mathrm{C}$ at the wobble site of a tRNA ${ }^{\text {Ile }}$ anticodon, i.e., we can disregard $\mathrm{M}_{\mathrm{wC}}$. If nature has chosen $\mathrm{G}$ at the wobble site of tRNA ${ }^{\text {Ile }}$ anticodon, then we may infer that $\mathrm{M}_{\mathrm{wG}}$ is the smallest. From $\mathrm{M}_{\mathrm{wG}}<\mathrm{M}_{\mathrm{wA}}$ and $\mathrm{M}_{\mathrm{wG}}<\mathrm{M}_{\mathrm{wU}}$ we have

$$
\begin{aligned}
& \frac{M_{U 3: G}}{M_{O}}<\frac{N_{C}}{N_{U}} \\
& N_{U} M_{U 3: G}<\left(N_{C}+N_{U}\right) M_{X 3: U}-N_{A} M_{O} \\
& \frac{M_{U 3: G}}{M_{Y 3: U}}<\frac{N_{C}+N_{U}-N_{A}}{N_{U}} \text {, assuming } M_{Y 3: U}<M_{O}
\end{aligned}
$$

where the assumption is made on the basis of previous observations that $U$ is generally the most versatile in wobble-pairing among the four nucleotides [7-12].

\section{Four-fold NNN codons}

Designate the number of codons ending with $A, C, G$, and $\mathrm{U}$ as $\mathrm{N}_{\mathrm{A}^{\prime}} \mathrm{N}_{\mathrm{C}^{\prime}} \mathrm{N}_{\mathrm{G}^{\prime}}$ and $\mathrm{N}_{\mathrm{U}^{\prime}}$, respectively. The wobble costs involving an $\mathrm{A}, \mathrm{C}, \mathrm{G}$ or $\mathrm{U}$ at the wobble site of tRNA anticodon are, respectively,

$$
\begin{aligned}
& M_{w A}=N_{A} M_{A 3: A}+N_{C} M_{C 3: A}+N_{G} M_{G 3: A}=\left(N_{A}+N_{C}+N_{G}\right) M_{O} \\
& M_{w C}=N_{A} M_{A 3: C}+N_{C} M_{C 3: C}+N_{U} M_{U 3: C}=\left(N_{A}+N_{C}\right) M_{O}+N_{U} M_{U 3: C} \\
& M_{w G}=N_{A} M_{A 3: G}+N_{G} M_{G 3: G}+N_{U} M_{U 3: G}=\left(N_{A}+N_{G}\right) M_{O}+N_{U} M_{U 3: G} \\
& M_{w U}=N_{C} M_{C 3: U}+N_{G} M_{G 3: U}+N_{U} M_{U 3: U}=\left(N_{C}+N_{U}\right) M_{Y 3: U}+N_{G} M_{G 3: U}
\end{aligned}
$$

If nature has chosen a $U$ at the wobble site of the tRNA anticodon, then Eq. (8) does not give us any simple inequality to estimate the cost ratios. However, if nature has chosen an $\mathrm{A}$ at the wobble site of the tRNA anticodon, then from $\mathrm{M}_{\mathrm{wA}}<\mathrm{M}_{\mathrm{wG}}$ and $\mathrm{M}_{\mathrm{wA}}<\mathrm{M}_{\mathrm{wC}}$, we can infer

$$
\begin{aligned}
\frac{M_{U 3: G}}{M_{O}}>\frac{N_{C}}{N_{U}} \\
\frac{M_{U 3: C}}{M_{O}}>\frac{N_{G}}{N_{U}}
\end{aligned}
$$

In what follows, we estimate $\mathrm{M}_{\mathrm{U} 3: \mathrm{G}} / \mathrm{M}_{\mathrm{O}}, \mathrm{M}_{\mathrm{G} 3: \mathrm{U}} / \mathrm{M}_{\mathrm{O}}$, and $\mathrm{M}_{\mathrm{Uz}: \mathrm{C}} / \mathrm{M}_{\mathrm{O}}$ by using fungal mitochondrial genomic data. Cells in fungal species are generally rapid-replicating which necessitates efficient translation. Rapidly replicating unicellular organisms are theoretically expected to be under strong selection to increase the rate of biosynthesis $[15,28]$ and they typically exhibit strong codon-anticodon adaptation [29]. Thus, fungal species should be ideal for evaluating evolutionary hypothesis on codon-anticodon adaptation.

\section{Methods}

We retrieved 36 fungal mitochondrial genomes (Table 1) by using NCBI Entrez. Three different genetic codes are used in different fungal genomes. Among the 36 fungal mitochondrial genomes, seven genomes use translation table 3, 27 genomes use translation table 4, and two 
genomes use translation table 16 (Table 1). When results are similar among genomes using the same translation table, only results from a representative genome are presented. The number of codon families supporting CAAH $\left(\mathrm{N}_{\mathrm{CAAH}}\right)$ and WVH $\left(\mathrm{N}_{\mathrm{WVH}}\right)$ is compiled following the following rationale [27]. Suppose a lysine (Lys) codon family has 20 AAA and 60 AAG codons. WVH would ignore the codon usage bias and predict a wobble $U$ in the tRNALys anticodon because $U$ can pair with both $A$ and $G$, whereas CAAH would predict a wobble $\mathrm{C}$ in the tRNALys anticodon to maximize the Watson-Crick match with the more frequent G-ending codons. If the tRNA ${ }^{\text {Lys }}$ anticodon is found to have a wobble $\mathrm{U}$, then WVH is supported; if a wobble $\mathrm{C}$ is found, then $\mathrm{CAAH}$ is supported. If we have 60 AAA codons and 20 AAG codons and if tRNA ${ }^{\text {Lys }}$ anticodon has a wobble $\mathrm{U}$, then both hypotheses are supported, i.e., they are indistinguishable and are not included in Table 1. The methionine codon families are not included in Table 1 but discussed in detail elsewhere $[27,30]$.

The tRNA and CDS sequences were extracted and analyzed by using DAMBE [31,32]. The CDS-derived codon usage is also computed with DAMBE. The anticodon in almost all tRNA sequences from all species share the regular feature of being flanked by two nucleotides on either side to form a loop that is held together by a stem. For example, the anticodon loop (AC loop) of the tRNAArg genes translating CGN codons in Epidermophyton floccosum is 28CGUGUUACGGCCACG42, where the starting and ending numbers indicate the position of the AC loop in

Table I: Number of codon families unambiguously supporting the codon-anticodon adaptation hypothesis $\left(\mathbf{N}_{\mathrm{CAAH}}\right)$ and the wobble versatility hypothesis $\left(\mathbf{N}_{\mathrm{WVH}}\right)$ in each fungal species.

\begin{tabular}{|c|c|c|c|c|}
\hline Species & Accession* & Codet & $\mathbf{N}_{\text {CAAH }}$ & $\mathbf{N}_{\text {WVH }}$ \\
\hline Allomyces macrogynus & $\underline{N C 001715}$ & 4 & I & 13 \\
\hline Ashbya gossypii ATCC 10895 & NC 005789 & 3 & 0 & 10 \\
\hline Aspergillus niger & NC 007445 & 4 & 0 & 12 \\
\hline Aspergillus tubingensis & NC 007597 & 4 & 0 & 12 \\
\hline Candida albicans SC53 I 4 & NC 002653 & 4 & I & II \\
\hline Candida glabrata & NC 004691 & 3 & 0 & 12 \\
\hline Candida metapsilosis & NC 00697I & 4 & I & 15 \\
\hline Candida orthopsilosis & NC 006972 & 4 & 1 & 15 \\
\hline Candida parapsilosis & NC 005253 & 4 & I & 15 \\
\hline Candida stellata & NC 005972 & 4 & I & 13 \\
\hline Moniliophthora perniciosa & NC 005927 & 4 & 0 & 13 \\
\hline Epidermophyton floccosum & NC_007394 & 4 & 2 & 12 \\
\hline Harpochytrium sp. JEL94 & NC 004760 & 4 & I & 3 \\
\hline Harpochytrium sp. JEL I 05 & NC 004623 & 4 & I & 4 \\
\hline Hyaloraphidium curvatum & NC 003048 & 4 & I & 4 \\
\hline Hypocrea jecorina & NC 003388 & 4 & I & 13 \\
\hline Kluyveromyces lactis & NC 006077 & 4 & 0 & 13 \\
\hline Kluyveromyces thermotolerans & NC 006626 & 3 & 0 & II \\
\hline Lecanicillium muscarium & $\overline{N C \quad 0045 I 4}$ & 4 & 0 & 10 \\
\hline Monoblepharella sp. JEL I5 & $\underline{N C \quad 004624}$ & 4 & 0 & 15 \\
\hline Mortierella verticillate & NC 006838 & 4 & I & 13 \\
\hline Penicillium marneffei & NC 005256 & 4 & I & 12 \\
\hline Pichia Canadensis & NC 001762 & 4 & 2 & 12 \\
\hline Podospora anserine & NC 001329 & 4 & 0 & 13 \\
\hline Rhizophydium sp. 136 & NC 003053 & 16 & 0 & 2 \\
\hline Rhizopus oryzae & NC 006836 & 4 & 1 & 14 \\
\hline Saccharomyces cerevisiae & NC_001224 & 3 & I & 12 \\
\hline Saccharomyces castellii & NC 003920 & 3 & 0 & 14 \\
\hline Saccharomyces servazzii & NC 004918 & 3 & 0 & 9 \\
\hline Schizophyllum commune & NC 003049 & 4 & I & 13 \\
\hline Schizosaccharomyces japonicus & NC 004332 & 4 & 1 & 16 \\
\hline Schizosaccharomyces octosporus & NC 004312 & 4 & 1 & 14 \\
\hline Schizosaccharomyces pombe & NC 001326 & 4 & I & 14 \\
\hline Smittium culisetae & NC 006837 & 4 & I & 15 \\
\hline Spizellomyces punctatus I & NC 003052 & 16 & 0 & 4 \\
\hline Yarrowia lipolytica & NC 002659 & 3 & 0 & II \\
\hline Sum & & & 23 & 414 \\
\hline
\end{tabular}

* GenBank accession number.

† Genetic code. 
the tRNA sequence, with the anticodon 5'-ACG-3' (matching codon CGU) flanked by two nucleotides on either side (in bold) to form a loop that is held together by a stem made of the first and the last four nucleotides. Similarly, the other tRNAArg translating AGR codons is 25AAAAUACUUCUAAUAUUUU 43 , with the AC loop held together by a six-base stem. However, some tRNA sequences have a suspicious AC loop and DAMBE will flag them out. The AC loop is then identified by aligning the tRNA sequences against other isoaccepting tRNA sequences with a regular AC loop [6]. Some tRNA anticodon loop has the anticodon flanked by three instead of two nucleotides. For example, the anticodon loop in tRNA ${ }^{\text {Leu }}$ in the mitochondrial genome of Kluyveromyces thermotolerans is GAUACUCUUAAGAUGUAUU, with the anticodon UAA flanked by three nucleotides (in bold) on both sides. There are a few tRNA sequences in which anticodon loop cannot be identified.

Some mitochondrial genomes in GenBank are annotated incorrectly. For example, tRNA ${ }^{\text {Pro }}$ in the mitochondrial genome of Ashbya gossypii ATCC 10895 has an anticodon of UGG matching codon CCA (the most frequently used proline codon), but the GenBank file (NC_005789) annotated the anticodon to match codon CCU.

A few fungal mitochondrial genomes do not have a complete set of tRNA genes. For example, the mitochondrial genomes of Hyaloraphidium curvatum and Harpochytrium $s p$. JEL94 have seven and eight tRNA genes, respectively, and consequently will need tRNA import from the nuclear genome. This may cause complication in analyzing codon-anticodon adaptation. However, removing such genomes does not alter the conclusions.

Some species exhibit extreme avoidance of certain codon families. For example, Ashbya gossypii ATCC 10895 codes Arg with only AGR codons without using any CGN codons. In contrast, Hyaloraphidium curvatum codes Arg with only CGN codons without using any AGR codons. Such avoidance of certain codon families would facilitate the evolutionary loss of the associated tRNA [33-35], although it is not always clear whether the avoidance is the cause or the consequence of the loss of the associated tRNA.

We computed relative synonymous codon usage, or RSCU [36], as a measure of codon usage bias within a codon family by using DAMBE [31,32]. Some coding sequences are incomplete. For example, the cox1 CDS in Aspergillus niger is annotated as "join(<19768..20614,21640..22495)". The first two nucleotides (i.e., at positions 19768 and 19769) represent a partial codon and are discarded in computing codon frequencies.

\section{Results and discussion \\ Wobble cost between G and U: MU3:G and MG3:U}

Recall that the two inequalities

$$
\begin{aligned}
\frac{M_{U 3: G}}{M_{O}}<\frac{N_{C}}{N_{U}} \text { and } \\
\frac{M_{G 3: U}}{M_{O}}<\frac{N_{A}}{N_{G}}
\end{aligned}
$$

are, respectively, for NNY codons translated by tRNA with a G at the wobble site of tRNA anticodon, and for NNR codons translated by tRNA with a $U$ at the wobble site of tRNA anticodon. The observed $\mathrm{N}_{\mathrm{C}} / \mathrm{N}_{\mathrm{U}}$ ratios for Allomyces macrogynus (representing fungal mitochondrial genomes with translation table 4) are much smaller than $\mathrm{N}_{\mathrm{A}} / \mathrm{N}_{\mathrm{G}}$ ratios (Table 2). The smallest $\mathrm{N}_{\mathrm{C}} / \mathrm{N}_{\mathrm{U}}$ value is 0.279 whereas the smallest $\mathrm{N}_{\mathrm{A}} / \mathrm{N}_{\mathrm{G}}$ value is 2.372 (Table 2). We have mentioned before that, if $\mathrm{M}_{\mathrm{U} 3: \mathrm{G}}$ is very small, then a wobble $\mathrm{G}$ at the tRNA anticodon will not impose strong selection against $\mathrm{U}$-ending codons, and $\mathrm{N}_{\mathrm{U}}$ may drift up and down with mutation relative to $\mathrm{N}_{\mathrm{C}}$. This will lead to relatively small $\mathrm{N}_{\mathrm{C}} / \mathrm{N}_{\mathrm{U}}$ ratios. From the minimum $\mathrm{N}_{\mathrm{C}} / \mathrm{N}_{\mathrm{U}}$ value of 0.279 , we may infer that $\mathrm{M}_{\mathrm{U} 3: \mathrm{G}}<0.279 \bullet \mathrm{M}_{\mathrm{O}}$, i.e., $M_{U 3: G}$ is quite small relative to $M_{O}$.

The AUH codon family coding for amino acid Ile in A. macrogynus mitochondrial genome is translated by a tRNA with a GAU anticodon. According to Eq. (7), the $\mathrm{M}_{\mathrm{U}: \mathrm{G}} /$ Co ratio should also be smaller than the $\mathrm{N}_{C} / \mathrm{N}_{U}$ ratio. The observed $\mathrm{N}_{C} / \mathrm{N}_{\mathrm{U}}$ ratio is $0.3605(=159 / 441)$. This is similar to the $\mathrm{N}_{\mathrm{C}} / \mathrm{N}_{\mathrm{U}}$ ratio in NNY codon families (Table 2). Thus, the wobble cost of $\mathrm{M}_{\mathrm{U} 3 \mathrm{G}}$ relative to $\mathrm{M}_{\mathrm{O}}$ from the AUH codon family is similar to that derived from NNY codon families.

The CUN codon family coding for amino acid Leu in A. macrogynus mitochondrial genome is translated by a tRNA with an AAG anticodon. Note that no $\mathrm{A} \rightarrow \mathrm{I}$ conversion has been observed in mitochondria $[37,38]$ so Eq. (9) is applicable. According to Eq. (9), the $\mathrm{M}_{\mathrm{U} 3: \mathrm{G}} / \mathrm{Co}$ ratio should be greater than the $\mathrm{N}_{C} / \mathrm{N}_{U}$ ratio. The observed $\mathrm{N}_{C} / \mathrm{N}_{U}$ ratio is $0.1905(=48 / 252)$. Thus we have $\mathrm{M}_{\mathrm{U} 3: \mathrm{G}}>0.1905 \bullet \mathrm{M}_{\mathrm{O}}$. This inequality, together with the previous inequality of $\mathrm{M}_{\mathrm{U} 3: \mathrm{G}}<0.279 \bullet \mathrm{M}_{\mathrm{O}}$, leads to $0.1905 \bullet \mathrm{M}_{\mathrm{O}}<\mathrm{M}_{\mathrm{U} 3: \mathrm{G}}<$ $0.279 \bullet \mathrm{M}_{\mathrm{O}}$.

Given that $\mathrm{M}_{\mathrm{U}: \mathrm{G}}>0.1905 \bullet \mathrm{M}_{\mathrm{O}}$, we can infer that $\mathrm{M}_{\mathrm{wG}}>$ $\mathrm{M}_{\mathrm{wA}}$ when $\mathrm{N}_{\mathrm{C}} / \mathrm{N}_{\mathrm{U}}<0.1905$, i.e., when U-ending codons are more than five times as frequent as $\mathrm{C}$-ending codons. In other words, when $\mathrm{N}_{\mathrm{C}} / \mathrm{N}_{\mathrm{U}}<0.1905$, the wobble cost of having an A at the wobble site of the tRNA anticodon is smaller than that of having a $G$ at the wobble site, and should be favored by natural selection. Among the 36 fungal mitochondrial genomes, only two genomes have a NNY codon family with a correspondent tRNA that has an 
Table 2: $\mathbf{N}_{C} / \mathbf{N}_{U}$ ratios for NNY codons and $\mathbf{N}_{\mathrm{A}} / \mathbf{N}_{\mathrm{G}}$ ratios for $\mathrm{NNR}$ codons in Allomyces macrogynus (representing fungal mitochondrial genomes with translation table 4).

\begin{tabular}{|c|c|c|c|c|c|c|c|c|c|}
\hline $\mathrm{CF}^{*}$ & $\mathrm{AA}^{\dagger}$ & $A C \ddagger$ & $\mathrm{N}_{\text {Cod }} \S$ & $\mathrm{N}_{\mathrm{C}} / \mathrm{N}_{\mathrm{U}}$ & CF* & $\mathrm{AA}^{\dagger}$ & $\mathrm{AC} \ddagger$ & $\mathrm{N}_{\text {Cod }} \S$ & $\mathrm{N}_{\mathrm{A}} / \mathrm{N}_{\mathrm{G}}$ \\
\hline AAY & $N$ & GUU & 360 & 0.295 & AAR & $\mathrm{K}$ & UUU & 411 & 2.543 \\
\hline AGY & $S$ & GCU & 230 & 0.314 & AGR & $\mathrm{R}$ & UCU & 202 & 6.769 \\
\hline CAY & $\mathrm{H}$ & GUG & 213 & 0.357 & CAR & $\mathrm{Q}$ & UUG & 173 & 3.806 \\
\hline GAY & $D$ & GUC & 356 & 0.299 & GAR & $\mathrm{E}$ & UUC & 263 & 2.372 \\
\hline UAY & $Y$ & GUA & 390 & 0.279 & UUR & L & UAA & 742 & 5.870 \\
\hline UGY & C & GCA & 94 & 0.382 & & & & & \\
\hline UUY & $\mathrm{F}$ & GAA & 592 & 0.726 & & & & & \\
\hline Minimum & & & & 0.279 & & & & & 2.372 \\
\hline Mean & & & & 0.379 & & & & & 4.272 \\
\hline Std Dev & & & & 0.157 & & & & & 1.975 \\
\hline
\end{tabular}

* CF: Codon family

t AA: One-letter code of amino acid

$\ddagger$ AC: Anticodon

$\S \mathrm{N}_{\text {Cod }}$ : Number of codons in the codon family

A at the wobble site of its anticodon. In the mitochondrial genome of Penicillium marneffei, the tRNA translating the AAY codon family has a wobble A in its anticodon. The $\mathrm{N}_{\mathrm{C}} / \mathrm{N}_{\mathrm{U}}$ ratio is $0.087(=41 / 473)$ which is much smaller than 0.1905. Similarly, in the mitochondrial genome of Pichia Canadensis, the tRNA translating the AGY codon family has a wobble $A$ in its anticodon. The $\mathrm{N}_{\mathrm{C}} / \mathrm{N}_{\mathrm{U}}$ ratio is $0.0083(=1 / 120)$ which is also much smaller than 0.1905 . Thus, the prediction that a wobble A at the tRNA anticodon is advantageous over a wobble $\mathrm{G}$ when $\mathrm{N}_{\mathrm{C}} / \mathrm{N}_{\mathrm{U}}$ $<0.1905$ is consistent with empirical data.

In contrast to the small $\mathrm{N}_{\mathrm{C}} / \mathrm{N}_{\mathrm{U}}$ ratios in $\mathrm{NNY}$, $\mathrm{AUH}$ and CUN codon families, all $\mathrm{N}_{\mathrm{A}} / \mathrm{N}_{\mathrm{G}}$ ratios in NNR codon families are substantially larger (Table 2 ). We have argued before that, if $\mathrm{M}_{\mathrm{G} 3: \mathrm{U}}$ is very small, then a $\mathrm{U}$ at the wobble site of tRNA anticodon would impose little selection against G-ending codons in NNR codon families, and mutation may allow $\mathrm{N}_{\mathrm{G}}$ to drift up, leading to large $\mathrm{N}_{\mathrm{G}}$ values relative to $\mathrm{N}_{A}$. However, if $\mathrm{M}_{\mathrm{G} 3 \mathrm{U}}$ is large, then $\mathrm{G}$ ending codons should be strongly selected against and $\mathrm{N}_{\mathrm{G}}$ would be small relative to $\mathrm{N}_{\mathrm{A}^{\prime}}$ leading to large $\mathrm{N}_{\mathrm{A}} / \mathrm{N}_{\mathrm{G}}$ ratios. The much larger $\mathrm{N}_{\mathrm{A}} / \mathrm{N}_{\mathrm{G}}$ ratios than $\mathrm{N}_{\mathrm{C}} / \mathrm{N}_{\mathrm{U}}$ ratios ( $\mathrm{t}$ $=5.2967, \mathrm{DF}=10, \mathrm{p}=0.0003$, two-tailed test) suggest that $\mathrm{M}_{\mathrm{G} 3: \mathrm{U}}$ is much greater than $\mathrm{M}_{\mathrm{U} 3: \mathrm{G}}$.

There is a caveat in evaluating the relative magnitude of $\mathrm{M}_{\mathrm{U} 3: \mathrm{G}} / \mathrm{M}_{\mathrm{O}}$, and $\mathrm{M}_{\mathrm{G} 3: \mathrm{U}} / \mathrm{M}_{\mathrm{O}}$ by the $\mathrm{N}_{\mathrm{C}} / \mathrm{N}_{\mathrm{U}}$ and $\mathrm{N}_{\mathrm{A}} / \mathrm{N}_{\mathrm{G}}$ ratios because these ratios can be affected by AT-biased mutations. The mitochondrial genome of $A$. macrogynus is $57472 \mathrm{bp}$, with the number of $\mathrm{C}+\mathrm{G}$ being 22700 and that of $\mathrm{A}+\mathrm{U}$ being 34772 . If we exclude those nucleotides in coding sequences, then the numbers of $\mathrm{C}+\mathrm{G}$ and $\mathrm{A}+\mathrm{U}$ are 14136 and 17656, respectively. This may be considered as the background frequencies maintained by mutation bias, which leads to the expected $\mathrm{N}_{\mathrm{C}} / \mathrm{N}_{\mathrm{U}}$ ratio of 0.8001
$\left(=\mathrm{N}_{\mathrm{C}+\mathrm{G}} / \mathrm{N}_{\mathrm{A}+\mathrm{U}}\right)$ and that of $\mathrm{N}_{\mathrm{A}} / \mathrm{N}_{\mathrm{G}}$ ratio of $1.2490\left(=\mathrm{N}_{\mathrm{A}+\mathrm{U}} /\right.$ $\mathrm{N}_{\mathrm{C}+\mathrm{G}}$ ). Thus, we expect $\mathrm{N}_{\mathrm{C}} / \mathrm{N}_{\mathrm{U}}$ to be smaller than $\mathrm{N}_{\mathrm{A}} / \mathrm{N}_{\mathrm{G}}$ even when there is no difference between $\mathrm{M}_{\mathrm{U} \text { :G }}$ and $\mathrm{M}_{\mathrm{G} 3: \mathrm{U}}$. To establish the argument that $\mathrm{M}_{\mathrm{U} 3: \mathrm{G}}$ is indeed smaller than $\mathrm{M}_{\mathrm{G} 3 \cdot \mathrm{U}}$, we need to demonstrate that (1) there is no selection against U-ending codons in NNY codon families by showing that the observed $\mathrm{N}_{C} / \mathrm{N}_{U}$ ratio is not greater than 0.8001 , and (2) there is selection against Gending codons in NNR codon families by showing that $\mathrm{N}_{\mathrm{A}} / \mathrm{N}_{\mathrm{G}}$ is significantly greater than 1.2490 . It is not enough to show that $\mathrm{N}_{\mathrm{C}} / \mathrm{N}_{\mathrm{U}}<\mathrm{N}_{\mathrm{A}} / \mathrm{N}_{\mathrm{G}}$.

We note that the observed $\mathrm{N}_{C} / \mathrm{N}_{\mathrm{U}}$ values for the seven NNY codon families in Table 2 are all smaller than the expected 0.8001 , suggesting no selection against U-ending codons in NNY codon families (i.e., small $\mathrm{M}_{\mathrm{U} 3: \mathrm{G}}$ ). In contrast the observed $\mathrm{N}_{\mathrm{A}} / \mathrm{N}_{\mathrm{G}}$ ratios for the five NNR codon families are all much greater than the expected 1.2490 (Table 2), consistent with the interpretation of selection against G-ending codons in NNR codon families (i.e., large $\left.\mathrm{M}_{\mathrm{G} 3: \mathrm{U}}\right)$. This is consistent with the interpretation that $\mathrm{M}_{\mathrm{U} 3: \mathrm{G}}<\mathrm{M}_{\mathrm{G} 3: \mathrm{U}}$. One can perform a $\chi^{2}$-test for each of the NNR codon families to see if G-ending codons are underused. The tests are all highly significant, with $\mathrm{p}<0.00001$.

The results are similar for fungal genomes with translation table 3, with the result from a representative species (Saccharomyces cerevisiae) presented in Table 3 . Again the $\mathrm{N}_{C} /$ $\mathrm{N}_{\mathrm{U}}$ ratios in NNY codon families are much smaller than $N_{A} / N_{G}$ ratios in NNR codon families. We should note that the $S$. cerevisiae mitochondrial genome is much more ATbiased than the A. macrogynus mitochondrial genome, with the proportion of $(\mathrm{G}+\mathrm{C})$ in non-coding sequences being only 0.1484 . The reason for the GC deficiency in yeast is not clear, but it may be caused either by mutation bias or by the low abundance of C in living cells [39-41]. 
In any case, the expected $\mathrm{N}_{\mathrm{C}} / \mathrm{N}_{\mathrm{U}}$ and $\mathrm{N}_{\mathrm{A}} / \mathrm{N}_{\mathrm{G}}$ ratios, given the biased genomic AT content, are 0.1742 and 5.7405, respectively. We note that the observed $\mathrm{N}_{\mathrm{C}} / \mathrm{N}_{\mathrm{U}}$ ratios among the NNY codon families are all smaller than the expected value of 0.1742 except for the UUY (Phe) codon family (Table 3), against suggesting little selection against U-ending codons (i.e., small $\mathrm{M}_{\mathrm{U} 3: \mathrm{G}}$ ). In contrast, the $\mathrm{N}_{\mathrm{A}} /$ $\mathrm{N}_{\mathrm{G}}$ ratios are all significantly greater than the expected 5.7405 except for the AUR (Met) codon family, suggesting selection against $\mathrm{G}$-ending codons (i.e., large $\mathrm{M}_{\mathrm{G} 3: \mathrm{U}}$ ). The exceptional AUR (Met) codon family has a tRNA with a CAU anticodon which would favor G-ending codons and is expected to be different.

The $\mathrm{N}_{\mathrm{C}} / \mathrm{N}_{\mathrm{U}}$ ratio for the UUY (Phe) codon family is consistently greater than those of other NNY codon families (Tables 2,3). One may suspect whether, for this particular codon family, there is a significant $\mathrm{M}_{\mathrm{U} 3 \mathrm{G}}$. The rate of tRNA ${ }^{\text {Phe }}$ with anticodon 3'-AAG-5' dissociating from the UUU codon is about twice as high as that from the fully matched UUC codon [42]. Also, the tRNA ${ }^{\text {Phe misread }}$ CUC codons more than twice as often as CUU codons [42]. These two lines of evidence suggest that C3:G pair is much more favorable than U3:G pair, i.e., $M_{U 3: G}$ for the UUY (Phe) codon family may indeed be substantially greater than $\mathrm{M}_{\mathrm{C} 3: \mathrm{G}}$. Unfortunately, there has been no other similar studies on codon-anticodon pairing for other NNY codon families. One should also note that the tRNA ${ }^{\text {Phe }}$ in this study comes from Escherichia coli, and the result may not be applicable to fungal species.

The two fungal species using genetic table 16, i.e., Spizellomyces punctatus and Rhizophydium sp. 136 each have only a partial set of tRNA genes. Among NNY codon families in $S$. punctatus, only the GAY (coding for Asp) and UAY codon family (coding for Tyr) have an identifiable tRNA, with anticodons being GUC and GUA, respectively. The expected $\mathrm{N}_{\mathrm{C}} / \mathrm{N}_{\mathrm{U}}$ and $\mathrm{N}_{\mathrm{A}} / \mathrm{N}_{\mathrm{G}}$ ratios are 0.4536 and 2.2048, respectively, based on the nucleotide frequencies of noncoding sequences in the mitochondrial genome. The observed $\mathrm{N}_{\mathrm{C}} / \mathrm{N}_{\mathrm{U}}$ ratios for GAY and UAY codon families are $0.1667(=40 / 240)$ and $0.2411(=81 / 336)$, respectively, suggesting no selection against $\mathrm{U}$-ending codons (i.e., small $\mathrm{M}_{\mathrm{U} \text { :G }}$ ). In contrast, the $\mathrm{N}_{\mathrm{A}} / \mathrm{N}_{\mathrm{G}}$ ratios are much larger, being 19.2308 (= 250/13) for the AAR codon family (coding for amino acid Lys) translated by a tRNA with a UUU anticodon and $11.1538(=145 / 13)$ for the CAR codon family (coding for amino acid Gln) translated by a tRNA with a UUG anticodon. These results suggest selection against G-ending codons (i.e., large $\mathrm{M}_{\mathrm{G} 3: \mathrm{U}}$ ) There is no other NNR codon family with identifiable tRNAs in $S$. punctatus. The other species using translation table 16, Rhizophydium sp. 136, exhibits a similar pattern.

In short, results from fungal mitochondrial genomes are consistent with no selection against U-ending codons in NNY codon families but significant selection against Gending codons in NNR codon families, indicating that $\mathrm{M}_{\mathrm{U} 3: \mathrm{G}}$ is smaller than $\mathrm{M}_{\mathrm{G} 3: \mathrm{U}}$. These findings corroborate previous biochemical studies demonstrating that $\mathrm{U} 3: \mathrm{G}$ is energetically much more favorable than G3:U $[2,23-26]$. However, G3:U can be almost as good as A3:U when $\mathrm{U}$ is modified to $\mathrm{xm}^{5} \mathrm{U}[43]$.

The finding of a small $\mathrm{M}_{\mathrm{U} 3: \mathrm{G}}$ can explain puzzling observations in codon usage in fungal mitochondrial genomes. Take the tRNA ${ }^{\text {Ser }}$ translating the AGY codon family in the mitochondrial genome of Ashbya gossypii ATCC 10895 for example. The genome contains 31 AGU codons and no AGC codon. CAAH would have predicted an ACU antico-

Table 3: $\mathbf{N}_{C} / \mathbf{N}_{U}$ ratios for $N N Y$ codons and $\mathbf{N}_{A} / \mathbf{N}_{G}$ ratios for $N$ RR codons in Saccharomyces cerevisiae (representing fungal mitochondrial genomes with translation table 3 ).

\begin{tabular}{|c|c|c|c|c|c|c|c|c|c|}
\hline $\mathrm{CF}^{*}$ & $\mathrm{AA}^{\dagger}$ & $\mathrm{AC} \ddagger$ & $\mathrm{N}_{\operatorname{Cod}} \S$ & $\mathrm{N}_{\mathrm{C}} / \mathrm{N}_{\mathrm{U}}$ & $\mathrm{CF}^{*}$ & $\mathrm{AA}^{\dagger}$ & $A C \ddagger$ & $\mathrm{N}_{\text {Cod }} \S$ & $\mathrm{N}_{\mathrm{A}} / \mathrm{N}_{\mathrm{G}}$ \\
\hline AAY & $\mathrm{N}$ & GUU & 786 & 0.065 & AAR & K & UUU & 599 & 16.114 \\
\hline AGY & $S$ & GCU & 119 & 0.035 & AGR & $R$ & UCU & 225 & 36.500 \\
\hline AUY & I & GAU & 728 & 0.093 & AUR & $M$ & CAU & 428 & 1.326 \\
\hline CAY & $\mathrm{H}$ & GUG & 183 & 0.070 & CAR & Q & UUG & 165 & 8.706 \\
\hline GAY & $\mathrm{D}$ & GUC & 264 & 0.052 & GAR & $E$ & UUC & 220 & 9.000 \\
\hline UAY & $Y$ & GUA & 495 & 0.076 & UGR & W & UCA & 130 & 20.667 \\
\hline UGY & C & $\mathrm{GCA}$ & 92 & 0.070 & UUR & L & UAA & 911 & 64.071 \\
\hline UUY & $F$ & GAA & 445 & 0.369 & & & & & \\
\hline Minimum & & & & 0.035 & & & & & 1.326 \\
\hline Mean & & & & 0.104 & & & & & $22.34 I$ \\
\hline Std Dev & & & & 0.109 & & & & & 21.560 \\
\hline
\end{tabular}

* CF: Codon family

† AA: One-letter code of amino acid

$¥ \mathrm{AC}$ : Anticodon

$\S \mathrm{N}_{\text {Cod }}$ : Number of codons in the codon family 
don with perfect base pair with AGU codons, but the observed anticodon is GCU consistent with WVH. Such an anticodon makes sense only if $\mathrm{M}_{\mathrm{U3}: \mathrm{G}}$ is equal to $\mathrm{M}_{\mathrm{C} 3: \mathrm{G}^{\prime}}$ i.e., a wobble $\mathrm{G}$ in a tRNA anticodon can pair with $\mathrm{U}$ just as good as with $\mathrm{C}$ in the third codon position.

Can this finding be generalized to nuclear genomes and the translation machinery in the cytoplasm? If $\mathrm{M}_{\mathrm{U}: \mathrm{G}}$ is generally small, then we have an answer to a puzzling observation that has long baffled molecular evolutionary geneticists. The UGY codon family (coding for Cys) in the nuclear genome of $S$. cerevisiae is translated by tRNA

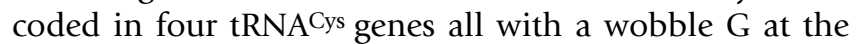
tRNA anticodon. One would have predicted that UGC codons should be strongly preferred over UGU codons to avoid the wobble cost $\mathrm{M}_{\mathrm{U3}: \mathrm{G}}$. However, the observed numbers of UGC and UGU codons in S. cerevisiae CDSs is 13802 and 22873, respectively, opposite to the predicted trend. The bias is even stronger in highly expressed genes. For example, in the codon usage table of highly expressed genes distributed with the EMBOSS package [[44], Eyeastcai.cut], the numbers of UGC and UGU codons are 3 and 39 , respectively. The unexpected codon usage bias (unexpected because the bias is in the wrong direction) becomes easy to understand if $\mathrm{M}_{\mathrm{U3:G}, \mathrm{G}}$ is equal to $\mathrm{M}_{\mathrm{C} 3: \mathrm{G}^{\prime}}$ i.e., there is no need to overuse the UGC codons to avoid $\mathrm{M}_{\mathrm{U} 3: \mathrm{G}}$ so AT-biased mutation in yeast (whose genome is highly AT-biased), which increases the frequency of Uending codons, is not checked by counteracting selection.

If the finding of $\mathrm{M}_{\mathrm{U3}: \mathrm{G}} \ll \mathrm{M}_{\mathrm{G} 3: \mathrm{U}}$ is applicable to nuclear genomes, then we predict that NNY codon families need only one type of tRNA (i.e., tRNA with a wobble $\mathrm{G}$ at the tRNA anticodon) for translation. In contrast, NNR codon families should ideally be translated by two different types of tRNAs, one with a wobble $U$ for NNA codons and the other with a wobble $\mathrm{C}$ for NNG codons (to avoid the relatively high wobble cost of $\mathrm{M}_{\mathrm{G} 3: \mathrm{U}}$ ). A corollary is that, if a NNY codon family is translated by only tRNA with a wobble $\mathrm{G}$ and NNR codon family by tRNA with a wobble $\mathrm{U}$, then codon usage bias should be smaller in the NNY codon family (in which selection against G-ending codon is weak because of the small $\mathrm{M}_{\mathrm{U}: \mathrm{G}}$ ) than in the NNR codon family (in which selection against U-ending codon is strong because of the relatively large $\mathrm{M}_{\mathrm{G} 3: \mathrm{U}}$ ). Below we test these predictions with genomic data.

\section{NNY codon families are translated by one type of tRNA with a wobble $G$ and NNR codon families are translated by two types of tRNA with a wobble $U$ and a wobble $C$, respectively, in fungal nuclear genomes}

We have inferred that a tRNA with a wobble $\mathrm{G}$ at its anticodon should be efficient not only in translating C-ending codons, but also in translating U-ending codons because of the small $\mathrm{M}_{\mathrm{U} 3: \mathrm{G}}$. For this reason, only one type of tRNA with a wobble $\mathrm{G}$ should generally be sufficient in translating NNY codon families. In contrast, in NNR codon families, tRNA with a wobble $U$ at its anticodon should be poor in translating G-ending codons because of the large $\mathrm{M}_{\mathrm{G} 3: \mathrm{U}}$. Thus, the presence of G-ending codons in NNR codon families should favour the use of two types of tRNAs, one with a wobble $U$ for translating NNA codons and another with a wobble $\mathrm{C}$ for translating NNG codons. In mitochondrial genomes with limited gene content, each codon family is generally translated by a single tRNA species. So this prediction cannot be tested. However, this prediction can be tested with nuclear genomes where the number of tRNA genes is not so limited as in mitochondrial genomes.

The prediction is strongly supported by results from the nuclear genome of Saccharomyces cerevisiae (Table 4). All NNR codon families are translated by two types of tRNAs with anticodons matching NNA and NNG codons, respectively, whereas all NNY codon families are translated by one type of tRNA with a wobble $G$ at the tRNA anticodon (Table 4). This is consistent with the interpretation that the inference of $\mathrm{M}_{\mathrm{U} 3: \mathrm{G}}<<\mathrm{M}_{\mathrm{G} 3: \mathrm{U}}$ derived from fungal mitochondrial genome is also applicable to fungal nuclear genomes.

One may propose an alternative hypothesis for the observation that NNY codon families are translated by tRNAs with a wobble $\mathrm{G}$ whereas NNR codon families translated by tRNAs with a wobble $\mathrm{C}$ and a wobble $\mathrm{U}$, respectively. The wobble A in some nuclear tRNAs is known to be converted to inosine or I for short [45-47] which may pair with A, C or U. If a tRNA translating an NNY codon family has its wobble $\mathrm{G}$ mutated to wobble $\mathrm{A}$, then the wobble A may undergo the $\mathrm{A} \rightarrow \mathrm{I}$ conversion and misread NNA codons. For this reason, the wobble $\mathrm{G} \rightarrow \mathrm{A}$ mutation should be strongly selected against, which would explain the lack of wobble A in tRNA translating NNY codons.

This alternative hypothesis invoking the $\mathrm{A} \rightarrow \mathrm{I}$ conversion, while logically sound, was dismissed quite early after the discovery $[45,47]$ that the $\mathrm{A} \rightarrow \mathrm{I}$ conversion is quite restrictive and occurs mainly at ACG or ARV anticodons (where $R$ is the IUB code for $A$ or $G$, and V is for $A, G$ or $C$ ). Among tRNAs translating NNY codons, only tRNA ${ }^{\text {Phe }}$ has a middle $\mathrm{A}$ in the anticodon, and the rest do not have an $\mathrm{R}$ in the middle of the anticodon. This means that, for all NNYtranslating tRNAs except for tRNA ${ }^{\text {Phe, }}$, even if their regular wobble $\mathrm{G}$ mutates to $\mathrm{A}$, the resulting wobble $\mathrm{A}$ will NOT be converted to inosine. Thus, the alternative hypothesis can only explain the avoidance of a wobble A in tRNA ${ }^{\text {Phe }}$ but cannot explain the avoidance of a wobble $\mathrm{A}$ in other NNY-translating tRNAs. 
Table 4: tRNAs translating two-fold codon families in the nuclear genome of Saccharomyces cerevisiae.

\begin{tabular}{llll}
\hline AA* & Codon & T & F \\
\hline Arg & AGA & 11 & 314 \\
Arg & AGG & 1 & 1 \\
Gln & CAA & 9 & 153 \\
Gln & CAG & 1 & 1 \\
Glu & GAA & 14 & 305 \\
Glu & GAG & 2 & 5 \\
Leu & UUA & 7 & 42 \\
Leu & UUG & 10 & 359 \\
Lys & AAA & 7 & 65 \\
Lys & AAG & 14 & 483 \\
Asn & AAC & 10 & 208 \\
Asn & AAU & 0 & 11 \\
Asp & GAC & 16 & 202 \\
Asp & GAU & 0 & 112 \\
Cys & UGC & 4 & 3 \\
Cys & UGU & 0 & 39 \\
His & CAC & 7 & 102 \\
His & CAU & 0 & 25 \\
Phe & UUC & 10 & 168 \\
Phe & UUU & 0 & 19 \\
Ser & AGC & 2 & 6 \\
Ser & AGU & 0 & 4 \\
Tyr & UAC & 8 & 141 \\
Tyr & UAU & 0 & 10 \\
& & &
\end{tabular}

* Amion acid carried by tRNA

t Codons forming Watson-Crick base pair with the anticodon of tRNA

$\ddagger$ Copy number of tRNA gene with anticodon matching the corresponding codon.

$\S$ Codon frequencies of highly expressed yeast protein-coding genes compiled in the Eyeastcai.cut file distributed with EMBOSS [44].

\section{Conclusion}

In summary, our general hypothesis based on wobble costs allows the integration of the two conventional hypotheses (i.e., CAAH and WVH) on codon-anticodon wobble pairing. The integration leads to new ways of evaluating relative wobble cost of different wobble pairings. In particular, the finding that $\mathrm{M}_{\mathrm{U}: \mathrm{G}}$ is much smaller than $\mathrm{M}_{\mathrm{G} 3: \mathrm{U}}$ corroborates previous structural studies showing that U3:G is energetically more favourable than G3:U and leads to a better understanding of the translation efficiency mediated by codon and anticodon wobble pairing.

\section{Acknowledgements}

This study is supported by the CAS/SAFEA International Partnership Program for Creative Research Teams and by NSERC's Discovery and Strategic Grants. I thank S. Aris-Brosou, P. Higgs and E. Rocha for discussion and comments and $E$. Westhof for references. Four anonymous reviewers provided helpful comments leading to substantial improvement of the paper.

\section{References}

I. Bonitz SG, Berlani R, Coruzzi G, Li M, Macino G, Nobrega FG, Nobrega MP, Thalenfeld $B E$, Tzagoloff $A$ : Codon recognition rules in yeast mitochondria. Proc Natl Acad Sci U S A 1980, 77(6):3167-3170.
2. Agris PF: Decoding the genome: a modified view. Nucleic Acids Res 2004, 32(I):223-238.

3. Tong KL, Wong JT: Anticodon and wobble evolution. Gene 2004, 333:169-I77.

4. Heckman JE, Sarnoff J, Alzner-DeWeerd B, Yin S, RajBhandary UL: Novel features in the genetic code and codon reading patterns in Neurospora crassa mitochondria based on sequences of six mitochondrial tRNAs. Proc Natl Acad Sci U S A 1980, 77(6):3I59-3163.

5. Martin RP, Sibler AP, Gehrke CW, Kuo K, Edmonds CG, McCloskey JA, Dirheimer G: 5-[[(carboxymethyl)amino]methyl]uridine is found in the anticodon of yeast mitochondrial tRNAs recognizing two-codon families ending in a purine. Biochemistry (Mosc) 1990, 29(4):956-959.

6. Xia X: Mutation and selection on the anticodon of tRNA genes in vertebrate mitochondrial genomes. Gene 2005, 345(1): 13-20.

7. Yokoyama S, Nishimura S: Modified nucleotides and codon recognition. In $t R N A$ : structure, biosynthesis and function Edited by: Soll D, RajBhandary U. Washington, ASM Press; 1995:207-223.

8. Sibler AP, Dirheimer G, Martin RP: Codon reading patterns in Saccharomyces cerevisiae mitochondria based on sequences of mitochondrial tRNAs. FEBS Lett 1986, 194(I): |3|-|38.

9. Inagaki Y, Kojima A, Bessho Y, Hori H, Ohama T, Osawa S: Translation of synonymous codons in family boxes by Mycoplasma capricolum tRNAs with unmodified uridine or adenosine at the first anticodon position. J Mol Biol I995, 25 I(4):486-492.

10. Yokobori S, Suzuki T, Watanabe K: Genetic code variations in mitochondria: tRNA as a major determinant of genetic code plasticity. J Mol Evol 200I, 53(4-5):3 I4-326.

II. Andachi Y, Yamao F, Iwami M, Muto A, Osawa S: Occurrence of unmodified adenine and uracil at the first position of anticodon in threonine tRNAs in Mycoplasma capricolum. Proc Natl Acad Sci U S A 1987, 84(2I):7398-7402.

12. Barrell BG, Anderson S, Bankier AT, de Bruijn MH, Chen E, Coulson AR, Drouin J, Eperon IC, Nierlich DP, Roe BA, Sanger F, Schreier PH, Smith AJ, Staden R, Young IG: Different pattern of codon recognition by mammalian mitochondrial tRNAs. Proc Natl Acad Sci U S A 1980, 77(6):3164-3166.

13. Bulmer M: Coevolution of codon usage and transfer RNA abundance. Nature 1987, 325(6 106):728-730.

14. Bulmer M: The selection-mutation-drift theory of synonymous codon usage. Genetics 199I, 129:897-907.

15. Xia $X$ : How optimized is the translational machinery in Escherichia coli, Salmonella typhimurium and Saccharomyces cerevisiae? Genetics 1998, 149(1):37-44.

16. Ikemura T: Correlation between the abundance of Escherichio coli transfer RNAs and the occurrence of the respective codons in its protein genes: a proposal for a synonymous codon choice that is optimal for the $E$ coli translational system. J Mol Biol I98I, I 5 I:389-409.

17. Gouy M, Gautier C: Codon usage in bacteria: correlation with gene expressivity. Nucleic Acids Res 1982, I0: 7055-7064.

18. Bennetzen JL, Hall BD: Codon selection in yeast. J Biol Chem I982, 257(6):3026-3031.

19. Akashi H: Translational selection and yeast proteome evolution. Genetics 2003, 164(4):1291-1303.

20. Eyre-Walker A: Synonymous codon bias is related to gene length in Escherichia coli: selection for translational accuracy? Mol Biol Evol 1996, 13(6):864-872.

21. Xia X: Maximizing transcription efficiency causes codon usage bias. Genetics 1996, 144:1309-1320.

22. Jia W, Higgs PG: Codon usage in mitochondrial genomes: distinguishing context-dependent mutation from translational selection. Mol Biol Evol 2008, 25(2):339-35I.

23. Ogle JM, Ramakrishnan V: Structural insights into translational fidelity. Annu Rev Biochem 2005, 74: I29-I77.

24. Mizuno H, Sundaralingam M: Stacking of Crick Wobble pair and Watson-Crick pair: stability rules of G-U pairs at ends of helical stems in tRNAs and the relation to codon-anticodon Wobble interaction. Nucleic Acids Res 1978, 5(I I):445I-446I.

25. Weixlbaumer A, Murphy FV, Dziergowska A, Malkiewicz A, Vendeix FA, Agris PF, Ramakrishnan V: Mechanism for expanding the decoding capacity of transfer RNAs by modification of uridines. Nat Struct Mol Biol 2007, I4(6):498-502. 
26. Masquida B, Westhof E: On the wobble GoU and related pairs. RNA 2000, 6(1):9-15.

27. Carullo M, Xia X: An Extensive Study of Mutation and Selection on the Wobble Nucleotide in tRNA Anticodons in Fungal Mitochondrial Genomes. J Mol Evol 2008, 66(5):484-493.

28. Xia X: Body temperature, rate of biosynthesis and evolution of genome size. Mol Biol Evol 1995, I 2:834-842.

29. Rocha EP: Codon usage bias from tRNA's point of view: Redundancy, specialization, and efficient decoding for translation optimization. Genome Res 2004, I 2: 12.

30. Xia X, Huang H, Carullo M, Betran E, Moriyama EN: Conflict between Translation Initiation and Elongation in Vertebrate Mitochondrial Genomes. PLoS ONE 2007, 2:e227.

31. Xia X, Xie Z: DAMBE: Software package for data analysis in molecular biology and evolution. J Hered 200I, 92(4):37I-373.

32. Xia X: Data analysis in molecular biology and evolution. Boston , Kluwer Academic Publishers; 2001:277.

33. Higgs PG, Jameson D, Jow $H$, Rattray M: The evolution of tRNALeu genes in animal mitochondrial genomes. J Mol Evol 2003 , 57(4):435-445.

34. Sengupta S, Higgs PG: A unified model of codon reassignment in alternative genetic codes. Genetics 2005, I 70(2):83I-840.

35. Sengupta S, Yang X, Higgs PG: The mechanisms of codon reassignments in mitochondrial genetic codes. J Mol Evol 2007, 64(6):662-688.

36. Sharp PM, Tuohy TM, Mosurski KR: Codon usage in yeast: cluster analysis clearly differentiates highly and lowly expressed genes. Nucleic Acids Res 1986, I 4(13):5 |25-5 I43.

37. Ling J, Roy $\mathrm{H}$, Qin D, Rubio MA, Alfonzo JD, Fredrick K, lbba M: Pathogenic mechanism of a human mitochondrial tRNAPhe mutation associated with myoclonic epilepsy with ragged red fibers syndrome. Proc Natl Acad Sci U S A 2007, 104(39): 15299-15304.

38. Grosjean H, Sprinzl M, Steinberg S: Posttranscriptionally modified nucleosides in transfer RNA: their locations and frequencies. Biochimie 1995, 77(1-2):|39-|4|.

39. Rocha EP, Danchin A: Base composition bias might result from competition for metabolic resources. Trends Genet 2002, I 8(6):29|-294.

40. Xia X, Palidwor G: Genomic Adaptation to Acidic Environment: Evidence from Helicobacter pylori. Am Nat 2005, 166(6):776-784

4I. Xia X, Wang HC, Xie Z, Carullo M, Huang H, Hickey DA: Cytosine usage modulates the correlation between CDS length and CG content in prokaryotic genomes. Mol Biol Evol 2006 , 23(7): 1450-I454.

42. Gromadski KB, Daviter T, Rodnina MV: A uniform response to mismatches in codon-anticodon complexes ensures ribosomal fidelity. Mol Cell 2006, 2 I(3):369-377.

43. Kurata S, Weixlbaumer A, Ohtsuki T, Shimazaki T, Wada T, Kirino Y, Takai K, Watanabe K, Ramakrishnan V, Suzuki T: Modified Uridines with C5-methylene Substituents at the First Position of the tRNA Anticodon Stabilize U\{middle dot\}G Wobble Pairing during Decoding. J Biol Chem 2008, 283(27):|880|-|88| I.

44. Rice P, Longden I, Bleasby A: EMBOSS: the European Molecular Biology Open Software Suite. Trends Genet 2000 16(6):276-277.

45. Haumont E, Fournier M, de Henau S, Grosjean H: Enzymatic conversion of adenosine to inosine in the wobble position of yeast tRNAAsp: the dependence on the anticodon sequence. Nucleic Acids Res 1984, I 2(6):2705-27I5.

46. Grosjean H, Auxilien S, Constantinesco F, Simon C, Corda Y, Becker HF, Foiret D, Morin A, Jin YX, Fournier M, Fourrey JL: Enzymatic conversion of adenosine to inosine and to $\mathrm{NI}$-methylinosine in transfer RNAs: a review. Biochimie 1996, 78(6):488-50I.

47. Auxilien S, Crain PF, Trewyn RW, Grosjean H: Mechanism, specificity and general properties of the yeast enzyme catalysing the formation of inosine $\mathbf{3 4}$ in the anticodon of transfer RNA. J Mol Biol 1996, 262(4):437-458.
Publish with Bio Med Central and every scientist can read your work free of charge

"BioMed Central will be the most significant development for disseminating the results of biomedical research in our lifetime. "

Sir Paul Nurse, Cancer Research UK

Your research papers will be:

- available free of charge to the entire biomedical community

- peer reviewed and published immediately upon acceptance

- cited in PubMed and archived on PubMed Central

- yours - you keep the copyright
BioMedcentral 